\title{
Bandwidth and Buffer Tradeoffs in Optical Packet Switching
}

\author{
Eric W. M. Wong, Senior Member, IEEE, and Moshe Zukerman, Senior Member, IEEE
}

\begin{abstract}
Recognizing the difficulties in buffering or slowing data in all-optical networks, optical packet switching (OPS) may be viable if buffers are small, at the cost of some inefficiency in link utilization. In this paper, the authors consider a new single-node OPS model that focuses on a set of output wavelengths in a certain link and consider the set of input wavelengths that transmits packets competing for the set of output wavelengths. Using this model, an exact solution and an accurate and scalable approximation, based on reduction of the state-space to a single direction, are provided for packet-loss probability and mean queueing delay. Tradeoffs between optical buffering capacity and link utilization for cases with and without wavelength conversion are studied and discussed.
\end{abstract}

Index Terms-All-optical networks, optical buffers, optical packet switching (OPS), packet-loss probability, queueing delay.

\section{INTRODUCTION}

$\mathbf{O}$ PTICAL packet switching (OPS) [1]-[12] has been proposed as a solution to overcome the "electronic bottleneck" [13] so that the future Internet can support ever-increasing traffic demands. OPS aims to achieve efficient all-optical networking by overcoming underutilization of optical circuit switching (OCS) [14]-[17] as well as potentially excessive packet loss in various optical burst switching (OBS) proposals [18]-[31]. OPS is based on buffering light which, despite the many advances, still remains a major impediment for successful OPS commercialization [32]-[34].

The transformation of the Internet from electronic packet switching to OPS exhibits a completely opposite cost structure. As pointed out in [35], [36], while in the electronic era, packet buffers have been cheap, long-haul transmission links have been expensive, optical buffering is expensive (and "painful"), and link capacity is "plentiful" with the ever-reducing cost per bit transmitted over optical links. This means that, while in the past, it was important to achieve high utilization on longhaul transmission links, in the optical era, it may be economical to design OPS networks with links running at, say, 50\%-70\%

Manuscript received June 8, 2006; revised September 13, 2006. This work was supported by a grant from the Research Grants Council of the Hong Kong Special Administrative Region, China under Project 9040928 and by the Australian Research Council.

E. W. M. Wong is with Department of Electronic Engineering, City University of Hong Kong, Hong Kong (e-mail: ewong@ee.cityu.edu.hk).

M. Zukerman is with the Australian Research Council Special Research Center for Ultra-Broadband Information Networks (CUBIN), Electrical and Electronic Engineering Department, The University of Melbourne, Victoria 3010, Australia (e-mail: mzu@unimelb.edu.au).

Color versions of Figs. 3-8 are available online at http://ieeexplore.ieee.org. Digital Object Identifier 10.1109/JLT.2006.885249

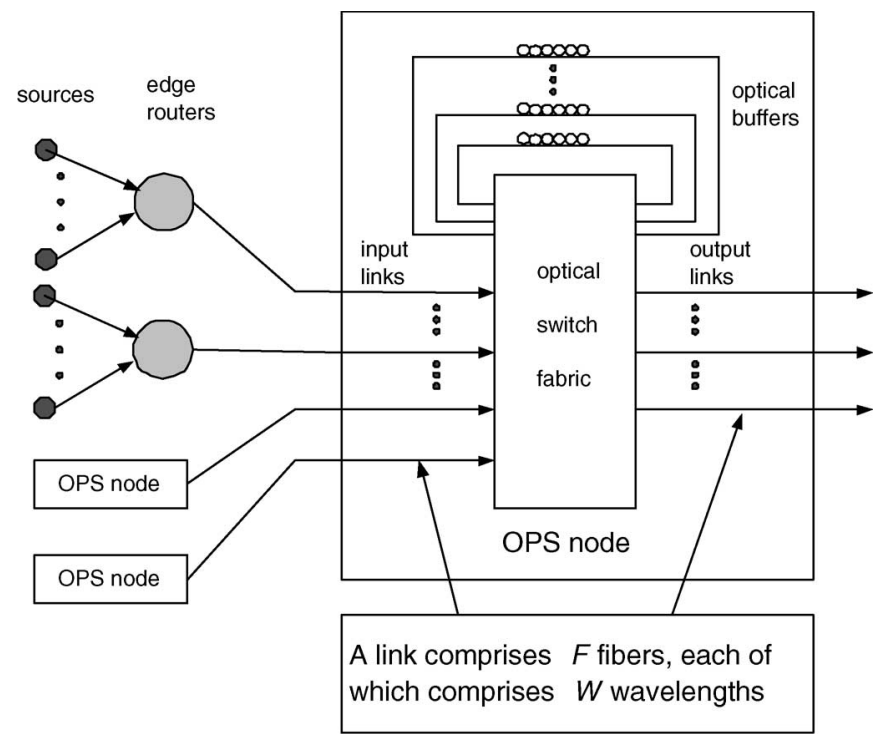

Fig. 1. OPS node model.

utilization or even lower, so that only small or even very small buffers are required.

These tradeoffs between optical buffering capacity and network utilization and the related cost evaluations are an important part of the economical analyses that may lead to decisions by vendors and telecommunication providers regarding their commitments to OPS. Using a relatively simple model based on a Poisson packet arrivals and modifying transmission control protocol (TCP), it is demonstrated in [35] and [36] that OPS can operate reasonably efficiently with very small buffers. They demonstrate by ns2 simulations [37] that a throughput of $70 \%-80 \%$ is achievable with buffers of $10-20$ packets. This is a significant step over previous publications [38], [39] that showed that buffers can be made smaller than the bandwidthdelay product but are still required to be a function of the bandwidth-delay product. Now, we have a demonstration that efficient operation is achievable with very small buffers and that their size is independent of the bandwidth-delay product.

Having recognized that OPS with very small buffers and less than $100 \%$ link utilization is an important option, we provide in this paper an analysis of an open-loop optical packet switch model to further study the tradeoff between buffer requirements and link utilization. In particular, we consider a nonblocking optical switch and focus our attention on a set of $K$ output wavelengths on a given link and consider a set of $N$ input wavelengths where packets are transmitted and compete for the $K$ output wavelengths. This is illustrated by Figs. 1 and 2. In Fig. 1, we describe a model of an OPS node 


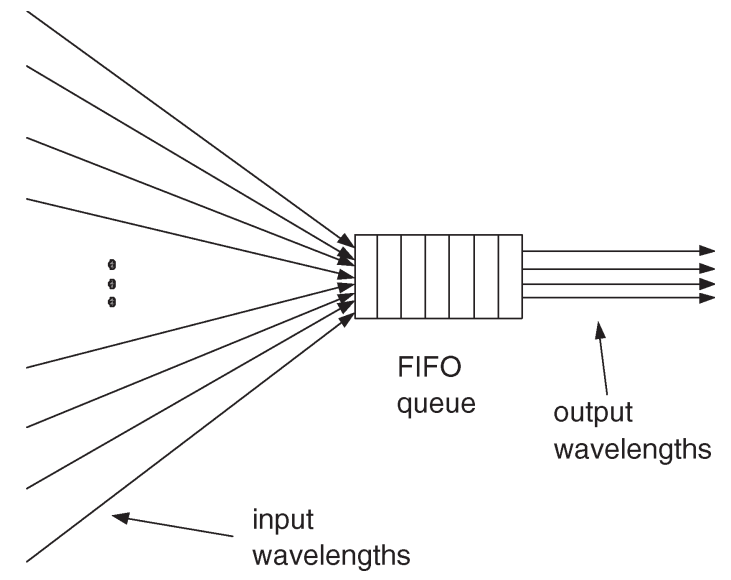

Fig. 2. Our node model.

where its input links can come from an edge router or other OPS nodes. Although the switch has many output ports (as seen in Fig. 1), the model is based on isolating one output port/link and all the input wavelengths through which packets are transmitted to that output link (see Fig. 2).

If all routes are unrestricted, the ratio $N / K$ is the number of input ports/links at the switch. If $N>K$ and if more than $K$ packets at the same time compete for the $K$ output wavelength, some packets may have to wait in a buffer. We assume that the buffer has a finite capacity, so a packet is dropped if it cannot find a wavelength and the buffer is full. To limit the use of the buffer, we further assume that no more than one packet from the same input wavelength is allowed in the buffer and that an arriving packet from a given input wavelength cannot be queued in the buffer if another packet from the same input wavelength is being transmitted in one of the $K$ output wavelengths. If this restriction is used in practice, it may avoid heavy users hogging the buffer during congestion.

We provide a scalable and accurate approximation for the packet blocking probability, the proportion of packets that are delayed, and the mean queueing delay of the packets that are delayed. The scalability is achieved by reducing the dimensionality of the state space to a single dimension using the fixed-point method. We also provide the procedure of obtaining the unique fixed-point solution and prove the convergence of the procedure. These scalable approximations can produce performance results in a fraction of a second; therefore, they can readily enable switch dimensioning for any case of realistic size. For a given traffic loading, we can compute the performance measures and increase the switch capacity until the required quality of service is obtained. Moreover, further extension of the single switch model presented here can be used as a module in a network-performance evaluation tool using fixed-point solutions as in [28]. Such a tool will enable network designers to decide on design parameters and evaluate cost.

The concept of "packet" in this paper does not have to be a single IP packet. It can also be a super-packet or a burst of many IP packets. As in OBS, many IP packets can be aggregated into a burst in an edge router and then each such burst can be transmitted individually over the all-optical OPS network. Such aggregation has the benefit of processing the header of an entire burst at each switch rather than individual IP packet headers. This also means that, if we say that we need a ten packet buffer, it possibly translates to buffer that may store 100s or 1000s IP packets.

Notice that having a finite $N$ is an important feature in OPS modeling. Notice that, if $N \leq K$, no loss will occur even if the buffer capacity is zero. However, analyses based on Poisson arrivals (which implies infinite $N$ ), as in [28] and [35], [36], will incorrectly show some losses for any finite-sized buffer. As will be demonstrated later, packet-loss probability based on our model are close to those obtained by Poisson assumption if $N \gg K$. However, in many situations, $N \gg K$ is not applicable, in which cases models such as the one proposed herein can be used.

Our model is somewhat similar to the well-known Engset model [40], but it is different in two ways. First, under the Engset model, when a packet is blocked, the "source" is immediately ready to transmit another packet. In our OPS model, the input wavelength is "frozen," and no other packet can be transmitted on it so long as the blocked packet is being dumped. Second, the Engset model assumes no buffer space. A model of a bufferless system that considers the effect of an input wavelength being "frozen" while a blocked packet is being dumped has been studied in [41]-[43], but these studies did not provide scalable approximations. Approximations for hybrid (OCS/OBS) systems that also considered the frozen input wavelength assumption are provided in [44]-[46].

It is known that full wavelength conversion is difficult and costly. Our model enables to consider cases with and without wavelength conversion by modifying the values of $N$ and $K$. An important question follows: Will small optical buffers enable a sufficient increase in link utilization, even without wavelength conversion, so that OPS can be economically justified? Using our model, we will provide numerical results that will shed some light on this question.

It is important to clarify that our model assumes that traffic is stationary. This is a common assumption in many analyses of telecommunications systems and networks, and it is usually made for tractability and simplicity. In fact, real traffic is far from being stationary. Designers usually use stationary traffic models by focusing on the busiest hour or the busiest minute of the day. If we assume our stationary traffic model, consider the busiest minute and decide on link capacities to meet, say, a blocking probability of 0.001 for a given buffer capacity and find that we need to run the link on, say, $75 \%$ utilization, but it does not mean that we achieve $75 \%$ utilization throughout the entire day. It may be much lower. Telecommunications providers may trade their excess capacity to maximize the efficiency of their networks, but this is beyond the scope of this paper. Here, we assume that traffic is stationary, keeping in mind that the utilization results we present are relevant to the busy period.

The remainder of the paper is organized as follows. In Section II, we present our single-node OPS model. In Section III, we provide a Markov chain analysis that leads to an exact solution. As the exact solution is not scalable, in Section IV, we provide a scalable approximation based on a fixed-point method and prove its convergence to a unique 
solution. In Section $\mathrm{V}$, we provide a range of numerical solutions that demonstrate the accuracy of the approximation, and we demonstrate performance results for cases with and without wavelength conversion. The conclusions are drawn in Section VI.

\section{NODE MODEL}

Consider an output link of an optical packet switch. The output link has $F$ optical fibers, and each optical fiber can carry $W$ wavelengths. If full wavelength conversion is available, this output link is viewed in our model as having $C=F W$ wavelengths. If no wavelength conversion is available, an arriving packet that uses a given wavelength must use the same wavelength at the output; thus, in this case, our output link is viewed, in our model, as having only $F$ wavelengths. Consider traffic flows coming from $N$ input wavelengths from various input links directed to an output link comprising $K$ wavelengths, which can take the values of $C$ or $F$ for the cases of with or without wavelength conversion, respectively. As in [44], we assume that the switching fabric is strictly nonblocking. There is no loss when $N \leq K$. Blocking probability computations only apply in the case of $(N>K)$, where loss may occur. Also, we assume that there is optical buffering capability (e.g., optical delay line) in the switch, such that packets can be stored optically. We assume that we can store up to $B$ packets in the switch. It is assumed that, as soon as one of the $K$ output wavelengths becomes free if the optical buffer is not empty, a packet from the optical buffer will be immediately transmitted on that output wavelength on a first come first served basis.

We assume that a packet is transmitted on a wavelength for an exponentially distributed period of time with mean $1 / \mu$. We consider the following ON-OFF process associated with each input wavelength. It is an alternating process where an OFF period follows an ON period, and an ON period follows an OFF period. A period of time used to transmit a single packet on an input wavelength is called an ON period, and the time between consecutive ON periods on that input wavelength is called an OFF period. The OFF period on an input wavelength is assumed to be exponentially distributed with mean $1 / \lambda$. This assumption of exponential times may not be as limiting as it may seem. It is well known that Engset formula [40] is insensitive to ON and OFF distributions [47]. The model we consider here has exponential times, but it has been shown that the burst blocking probability is not too sensitive to the distribution of the ON and OFF periods [41], [42], [44] in the bufferless case. Although the sensitivity of the blocking probability to the ON and OFF distributions is likely to increase with the buffer capacity $B$, it should not increase by too much due to the cost of optical buffering; $B$ is likely to be small.

As mentioned above, we further assume that no more than one packet from the same input wavelength is allowed in the buffer and that an arriving packet from a given input wavelength cannot be queued in the buffer, and it is therefore dumped if another packet from the same input wavelength is being transmitted in one of the $K$ output wavelengths. Note that these two assumptions are conservative. In addition, for tractability, we slightly strengthen the above two assumptions and assume that, if a packet from a given input wavelength is either buffered or transmitted, the input wavelength is considered "frozen" until its previous packet completes its transmission on an output wavelength. Notice that all this assumption is redundant in the cases of $B=0, N \rightarrow \infty$, and $K \gg B$. Following the "frozen" state period, the source will commence its OFF period as discussed above. In all other packet-loss events, namely, when all buffer places and all output wavelengths are occupied by packets from different input wavelengths and another packet from a new input wavelength arrives, this packet will be dumped. In such a case, no packet will arrive from the same input wavelength for the duration of the transmission of the dumped packet (a random variable with mean $1 / \mu$ ).

Figs. 1 and 2 illustrate the simplifications that take place in our model. Considering an optical switch with multiple input and multiple output ports, and optical buffering, we make two fundamental simplifications. First, we focus on a specific output link. This simplification is without loss of generality because we can repeat the modeling and the performance evaluation procedure for each of the output links. Second, we simplify the optical buffer to a first-in-first-out queue with the additional assumption of not having two packets in it from the same input wavelength. Note that, according to our network model, there are an edge router, where the possible bursty traffic stream is aggregated, and the optical switch, on which we focus our analysis, (both appear in Fig. 1). Although the edge router is subjected to bursty traffic loading which are electronically buffered there (possibly in very large buffers), its output has a smoothing effect on the traffic. In our analysis, we consider an optical switch in the core network where its traffic loading is not as bursty as the traffic loading on the edge router. A good example in illustrating this is by the aforementioned case of $N \leq K$, that is, if the number of input wavelengths of our optical switch is not higher than the number of its output wavelengths, the loss is zero, regardless of how bursty the original traffic is here and regardless of the variability in $\mathrm{ON}$ and OFF periods of the traffic in the input wavelength of our target switch.

\section{EXACT SOLUTION}

We will now present an exact solution for the model described in the previous section. Despite the assumption we have made for tractability, the exact solution we present here is still not scalable, and further approximations will be made in the next section.

Let $\pi_{i, j}(i, j \geq 0, i \leq K+B, j \leq N-K-B)$ be the probability that $i$ packets are being either transmitted or queued for transmission and $j$ input wavelengths are dumping blocked packets. Notice that, if a packet arrival (in the optical domain) is blocked and dumped, the input wavelength behaves as if it were inactive until the end of the packet has arrived at the switch. The number of idle input wavelengths is given by $N-i-j$. The $\pi_{i, j}$ values are obtained by the following steady state equations. For $i<K$

$$
\begin{aligned}
\pi_{i, j}((N-i-j) \lambda+ & (i+j) \mu)=\pi_{i-1, j}(N-i-j+1) \lambda \\
& +\pi_{i+1, j}(i+1) \mu+\pi_{i, j+1}(j+1) \mu
\end{aligned}
$$


and for $K \leq i \leq K+B$

$$
\begin{aligned}
\pi_{i, j} & ((N-i-j) \lambda+(K+j) \mu) \\
= & \pi_{i-1, j}(N-i-j+1) \lambda+\pi_{i, j-1} f(N-i-j+1) \lambda \\
\quad & +\pi_{i+1, j} K \mu+\pi_{i, j+1}(j+1) \mu
\end{aligned}
$$

where $f=1$ if $i=K+B$ and $=0$, otherwise. Introducing the normalization equation

$$
\sum_{\forall i, j} \pi_{i, j}=1
$$

gives rise to a set of equations, which can be used to obtain the $\pi_{i, j}$ probabilities. The total load offered by the packets is given by

$$
O=\sum_{\forall i, j}(N-i-j)(\lambda / \mu) \pi_{i, j}
$$

The total load carried by the packets is given by

$$
C=\sum_{\forall i<K+B, j}(N-i-j)(\lambda / \mu) \pi_{i, j}
$$

The average packet blocking probability $P_{B}$ is given by

$$
P_{B}=1-\frac{C}{O} .
$$

The utilization of the output link is given by

$$
U=\sum_{\forall i, j} \min (i, K) \pi_{i, j} .
$$

The carried load of the packets in the queue is

$$
\bar{Q}=\sum_{\forall K \leq i<K+B, j}(N-i-j)(\lambda / \mu) \pi_{i, j} .
$$

The proportion of the packets that are delayed, which is denoted as $P_{D}$, is

$$
P_{D}=\frac{\bar{Q}}{C} .
$$

The average number of packets in the system, which is denoted as $\bar{N}_{p}$, is given by

$$
\bar{N}_{p}=\sum_{\forall i, j} i \pi_{i, j} .
$$

By Little's formula, the mean packet delay (including queueing delay plus transmission time) is given by

$$
D=\frac{\bar{N}_{p}}{C \mu} .
$$

The mean packet queueing delay is given by

$$
\mathrm{QD}=D-1 / \mu \text {. }
$$

The mean queueing delay for a delayed packet, which is denoted as $\mathrm{QD}_{q}$, is given by

$$
\mathrm{QD}_{q}=\mathrm{QD} / P_{D}
$$

Given the exploding two-dimensional state-space, solving (1) and (2) is not scalable for large $K$ and $N$ values. A scalable approximation is derived next.

\section{APPROXIMATION}

The approximation is obtainable by a standard analysis of a single-dimension state-dependent multiserver finite-source finite-buffer queueing system. It is based on absorbing the state representing the number of dumped packets into a single state representing the total number of packets in the system, including both packets being served (transmitted by an output wavelength) and packets being queued.

In order to capture the effect of the dumped packets described by an additional "dumped" state $(j)$ in our exact solution, we consider the OFF period to be modified by the dumped packets. As in [44], we argue that, from the point of view of the switch, when a packet arrival is blocked and dumped, the input wavelength behaves as if it were inactive until the end of the packet has arrived at the switch. This can be considered equivalent to a situation whereby the blocked input wavelength having a longer OFF period with a mean equal to $1 / \mu+1 / \lambda$. This happens with probability $P_{B}$, which is the packet blocking probability calculated later on. Let $1 / \lambda^{*}$ be the modified mean OFF period; it is given by

$$
\frac{1}{\lambda^{*}}=\left(1-P_{B}\right) \frac{1}{\lambda}+P_{B}\left(\frac{1}{\mu}+\frac{1}{\lambda}\right)
$$

or

$$
\lambda^{*}=\frac{\mu}{\frac{\mu}{\lambda}+P_{B}} .
$$

Let $p_{i}$ be the probability that there are $i$ packets being either served or queued. This gives rise to a single-dimension birth-death process described by the following steady-state equations. For $1 \leq i \leq K$

$$
p_{i}=(N-i+1) \frac{\lambda^{*}}{i \mu} p_{i-1}
$$

and for $K<i \leq K+B$

$$
p_{i}=(N-i+1) \frac{\lambda^{*}}{K \mu} p_{i-1}
$$

and the normalization equation

$$
\sum_{i=0}^{K+B} p_{i}=1
$$

by which the $p_{i}$ values can be obtained. The total load offered by the packets is given by

$$
O=\sum_{i=0}^{K+B}(N-i)\left(\lambda^{*} / \mu\right) p_{i} .
$$

The total load carried by the packets is given by

$$
C=\sum_{i=0}^{K+B-1}(N-i)\left(\lambda^{*} / \mu\right) p_{i} .
$$


Therefore, the average packet blocking probability $P_{B}$ is given by

$$
P_{B}=1-\frac{C}{O}
$$

The utilization of the output link is given by

$$
U=\sum_{i=1}^{K+B} \min (i, K) p_{i} .
$$

The carried load of the packets in the queue is

$$
\bar{Q}=\sum_{i=K}^{K+B-1}(N-i)\left(\lambda^{*} / \mu\right) p_{i} .
$$

The proportion of the packets that are delayed, which is denoted as $P_{D}$, is

$$
P_{D}=\frac{\bar{Q}}{C}
$$

The average number of the packets in the system, which is denoted as $\bar{N}_{p}$, is given by

$$
\bar{N}_{p}=\sum_{i=1}^{K+B} i p_{i} .
$$

By Little's formula, the mean packets delay is given by

$$
D=\frac{\bar{N}_{p}}{C \mu} .
$$

The mean packet queueing delay is given by

$$
\mathrm{QD}=D-1 / \mu \text {. }
$$

The mean queueing delay for a delayed packet, which is denoted as $\mathrm{QD}_{q}$, is given by

$$
\mathrm{QD}_{q}=\mathrm{QD} / P_{D}
$$

Note that the functional relation between $\lambda^{*}$ expressed in (4) and $P_{B}$ expressed in (10) gives rise to a fixed-point equation. Next, we will prove that there must exist a unique fixed-point solution. Then, we will provide a binary search algorithm to find such a fixed-point solution and prove that such an algorithm converges to the desired unique fixed-point solution.

\section{A. Existence and Uniqueness of a Fixed Point}

For fixed values of $N, M, B$, and $\mu$, let the function $P_{B}(x)$ be the solution for $P_{B}$ obtained by (5)-(10) given that $x=\lambda^{*}$. Let the functions $f(x)$ and $g(x)$ be defined by

$$
f(x)=x
$$

and

$$
g(x)=\mu /\left(\mu / \lambda+P_{B}(x)\right)
$$

respectively. The fixed-point equation that we are solving is

$$
f(x)=g(x), \quad x>0 .
$$

Clearly, $f(x)$ is a monotonically increasing function of $x$. Also, $g(x)$ is a monotonically decreasing function of $x$ since $P_{B}(x)$ is a monotonically increasing function of $x$ (see the Appendix). In particular, $f(x)$ increases from zero to $\infty$, and $g(x)$ decreases from $\lambda$ to $\lambda \mu /(\lambda+\mu)$. Therefore, the two continuous curves must cross each other exactly once. It means that there must exist a unique fixed-point solution in the interval of $[\lambda \mu /(\lambda+$ $\mu), \lambda]$.

\section{B. Binary Search Algorithm for Finding the Fixed-Point Solution and Its Convergence}

Let the transformation from $\lambda(n)$ to $\lambda(n+1)$ (or $\lambda_{\text {previous }}$ to $\lambda_{\text {current }}$ in the following search algorithm) be defined by the function $\Gamma(x)$, where

$$
\Gamma(x)=g(x), \quad x \geq 0 .
$$

Let $\lambda^{*}$ be the unique fixed-point solution. Here is the binary search algorithm to find the fixed-point solution.

$$
\begin{aligned}
& \lambda^{+}=\lambda \\
& \lambda^{-}=\lambda \mu /(\lambda+\mu) \\
& \lambda_{\text {previous }}=\lambda^{+} \\
& \lambda_{\text {current }}=\lambda^{-} \\
& \text {while }\left|\lambda_{\text {current }}-\lambda_{\text {previous }}\right|>\epsilon \\
& \quad \lambda_{\text {previous }}=\lambda_{\text {current }} \\
& \lambda_{\text {current }}=\left(\lambda^{+}+\lambda^{-}\right) / 2 \\
& \text { if } \Gamma\left(\lambda_{\text {current }}\right)>\lambda_{\text {current }} \\
& \quad \lambda^{-}=\lambda_{\text {current }} \\
& \text { else } \\
& \quad \lambda^{+}=\lambda_{\text {current }} \\
& \text { end } \\
& \text { end }
\end{aligned}
$$

where $\epsilon$ is the error we can tolerate. Note that $\lambda^{+} \geq \lambda^{*} \geq \lambda^{-}$ and $\Gamma(x)$ [i.e., $g(x)$ ] is a monotonically decreasing function of $x$. Also note that

$$
\begin{aligned}
& \text { if } \lambda_{\text {current }}<\lambda^{*} \\
& \quad \Gamma\left(\lambda_{\text {current }}\right)>\lambda^{*} \\
& \Rightarrow \Gamma\left(\lambda_{\text {current }}\right)>\lambda_{\text {current }} \\
& \text { else } \\
& \quad \Gamma\left(\lambda_{\text {current }}\right)<\lambda^{*} \\
& \quad \Rightarrow \Gamma\left(\lambda_{\text {current }}\right)<\lambda_{\text {current }} .
\end{aligned}
$$

It means that

$$
\begin{aligned}
& \text { if } \Gamma\left(\lambda_{\text {current }}\right)>\lambda_{\text {current }} \\
& \lambda_{\text {current }}<\lambda^{*} \\
& \text { else } \\
& \quad \lambda_{\text {current }}>\lambda^{*} .
\end{aligned}
$$

It implies that, in the above algorithm, the interval of $\left[\lambda^{-}, \lambda^{+}\right]$always covers $\lambda^{*}$. It is also well known that binary 

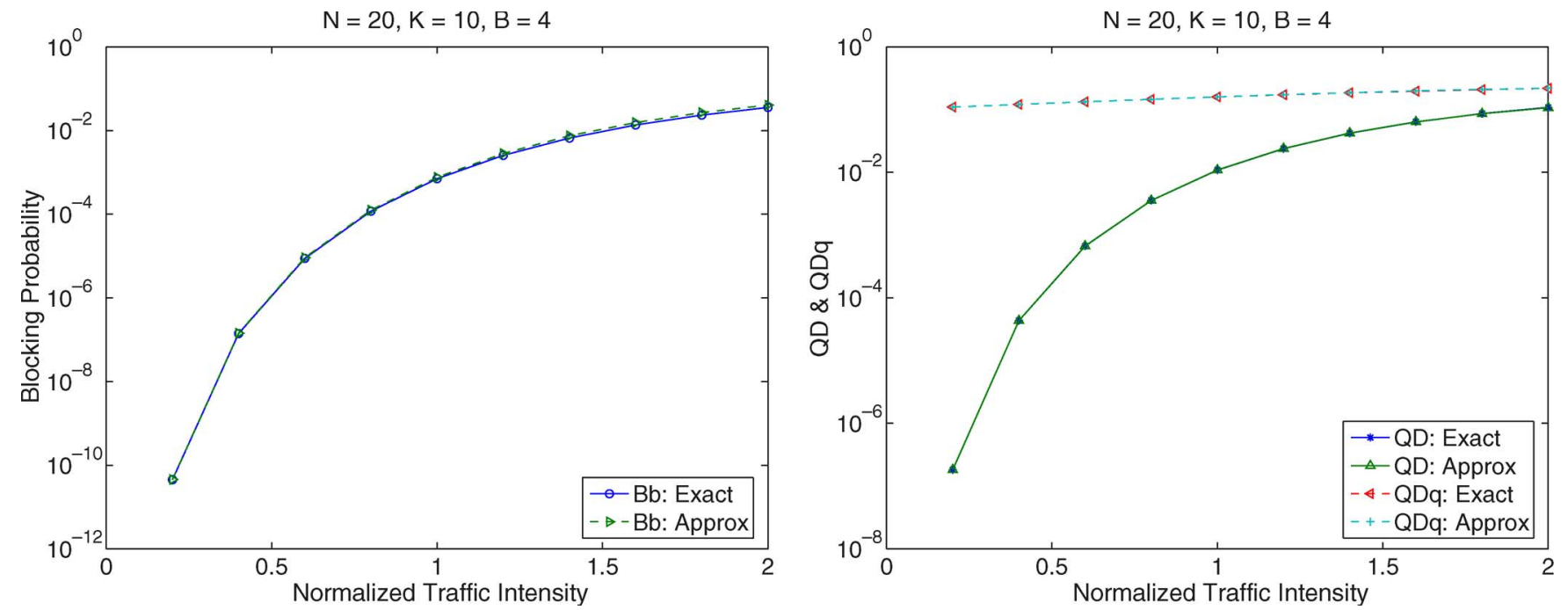

Fig. 3. Blocking probability (left) and delay [sec.] (right) versus normalized traffic intensity for $N=20, K=10$, and $B=4$.
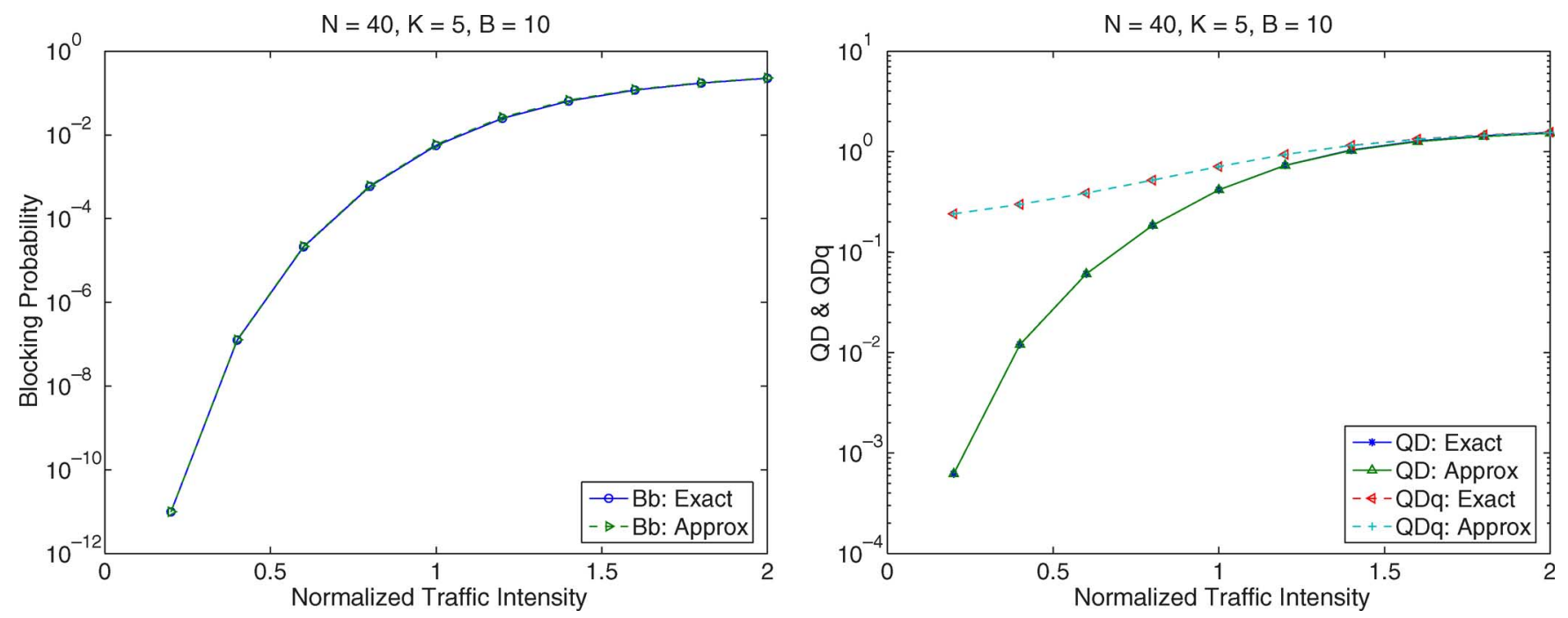

Fig. 4. Blocking probability (left) and delay [sec.] (right) versus normalized traffic intensity for $N=40, K=5$, and $B=10$.

search always converges to a single solution. Therefore, we can conclude that the proposed binary search algorithm converges to the desired unique fixed-point solution.

\section{Numerical RESUlts}

In this section, we present numerical results to evaluate the accuracy of our approximation and attempt to gain some insight into the important question of how far are we from OPS commercialization. We begin by quantifying, via exact solution obtained by (1) and (2), the accuracy of our approximations. Results are presented here for the blocking probability and mean queueing delay versus what we call the normalized traffic intensity, which is defined by $(N / K)(\lambda / \mu)$. In all our examples, we consider $\mu=1 \mathrm{~s}^{-1}$.

In all scenarios studied regardless of the values of $N, B$, $K$, and the traffic intensity, our numerical results show that the approximations agree very well with the exact solutions, as demonstrated, for example, in Figs. 3 and 4. The agreement demonstrated in Figs. 3 and 4 have also been observed in many other cases we considered; however, for brevity, we do not present them here. Note that it is possible that, during certain periods of time, the overall arrival rate increases beyond the service rate (e.g., because the number of possibly heavy loaded input wavelengths is higher than the number of output wavelengths), and it is interesting to evaluate the blocking probability in such situations. In addition, we consider here a loss system with a limited buffer space. Unlike an infinite buffer system, our system is still stable, even if the normalized traffic intensity is very large, and of course, this occurs at the expense of high blocking probability, as demonstrated in Figs. 3 and 4.

To compare between the cases of full and no wavelength conversions, we consider the case of $F=3$ and $W=40$, that is, each link carries three optical fibers with 40 wavelengths per fiber. Altogether, each link carries $3 \times 40=120$ wavelengths. Therefore, for the case of full wavelength conversion, we will have $K=120$, and an incoming packet on any input wavelength has the flexibility to choose any of the 120 wavelengths. For the case of no wavelength conversion, we will have $K=3$ because an incoming packet on a given input wavelength can 


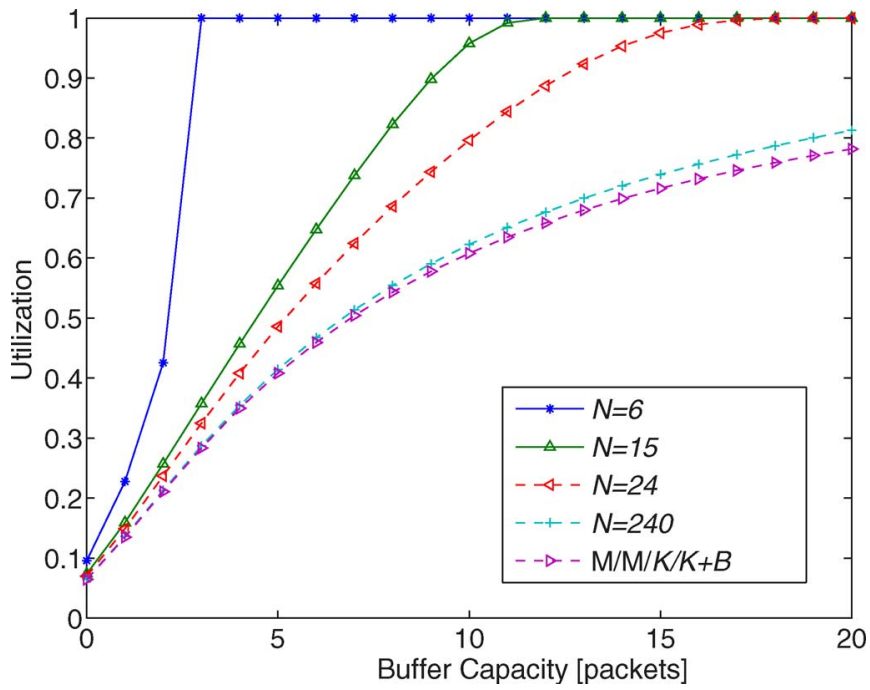

Fig. 5. Utilization versus buffer capacity [packets] for $K=3$, with $P_{B}=10^{-3}$.

choose only the same wavelength, and there are three such wavelengths on each of the fibers on the output link. The value of the relevant number of input wavelengths is set to be the number of input port times the relevant $K$. For example, $N=6$, for the case of no wavelength conversion, indicates two input ports.

Then, we consider the case of no wavelength conversion $(K=3)$ and a range of $N$ values and buffer capacity. For each case, using our results for packet blocking probability, we find the maximal $\lambda$ value, such that the blocking probability does not exceed $10^{-3}$. Then, we compute the utilization (of the output line) based on (11). For each $N$, we plot the utilization as a function of the buffer capacity [packets] in Fig. 5. Notice also that, in Fig. 5, we provide an additional curve based on an $\mathrm{M} / \mathrm{M} / K / K+B$ queue [48]. The $\mathrm{M} / \mathrm{M} / K / K+B$ queue assumes Poisson arrivals and exponential service times. It is equivalent to our model as $N \rightarrow \infty$. Notice that, for the case $N \rightarrow \infty$, the fact that a finite number of input wavelengths are "frozen" will not affect the Poisson nature of the arrival process. We can see that the results presented approach the $\mathrm{M} / \mathrm{M} / K / K+B$ curve as $N$ increases. This gives us additional confidence in our approximation as well as a conservative bound for the utilization. The results seem to be consistent with those of the study in [35] and [36], where OPS can operate reasonably efficiently with very small buffers even without wavelength conversion. However, it is worth noting that, without wavelength conversion, efficiency on routes with many hops in an all-optical network will be significantly reduced. In addition, considering our comments in the Introduction on the nonstationary of the traffic and the fact that we only design for the busiest minute or so, we can expect lower utilization in practice. Nevertheless, the nonstationarity is an issue with which we have always had to deal, regardless of whether the switching is electronic or optical.

In Fig. 6, we present equivalent results presented in Fig. 5, but here, we consider the case of full wavelength conversion $(K=120)$. As expected, the utilization is much higher, and reasonably efficient operation ( $70 \%$ utilization) can also

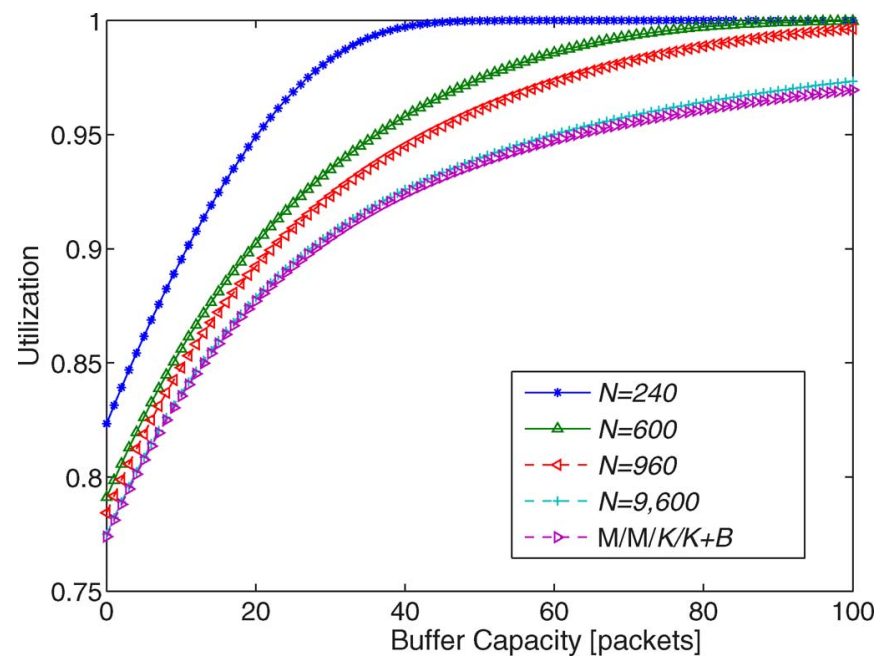

Fig. 6. Utilization versus buffer capacity [packets] for $K=120$, with $P_{B}=10^{-3}$

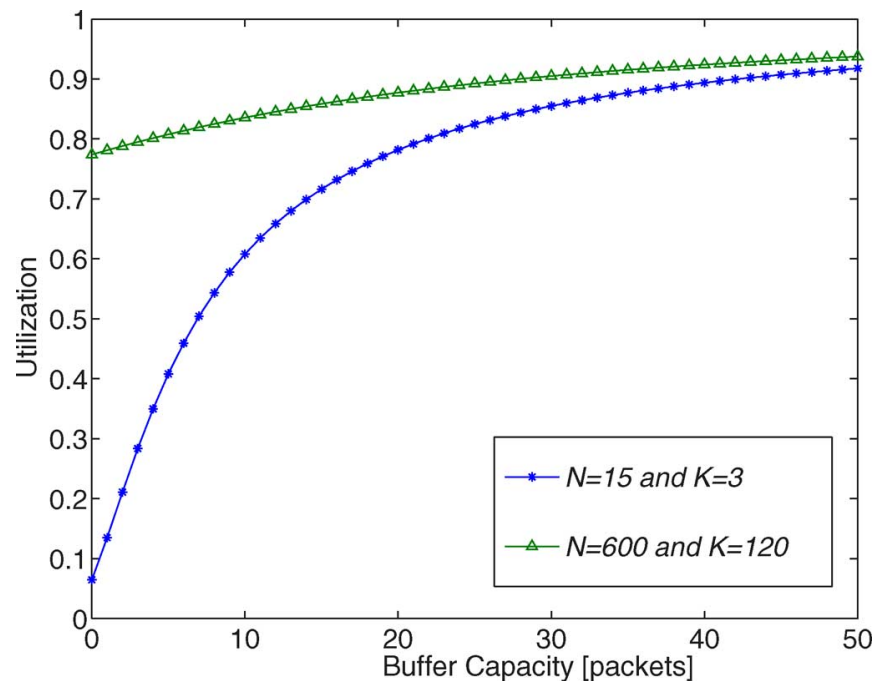

Fig. 7. Utilization versus buffer capacity [packets] for $\mathrm{M} / \mathrm{M} / K / K+B$, with $P_{B}=10^{-3}$.

be achieved in the bufferless case. Again, we see that the $\mathrm{M} / \mathrm{M} / K / K+B$ curve is consistent with the case $N \rightarrow \infty$.

The two $\mathrm{M} / \mathrm{M} / K / K+B$ curves of Figs. 5 and 6 are plotted next to each other in Fig. 7. We can see a clear benefit to full wavelength conversion in OPS for very low buffers, but the difference diminishes as the buffer capacity increases. For buffer capacity of 50 packets, both achieve more than $90 \%$ utilization.

One may argue against the blocking probability of $10^{-3}$, so we provide in Fig. 8 the equivalent results for blocking probability of $10^{-6}$. Now, we see that, without wavelength conversion, the link is almost empty in the bufferless case, but it increases fast with a small buffer and achieves over $70 \%$ utilization with a 50-packet buffer. Finally, we note that there are many options and variations of provision of limited wavelength conversion. Each of these options has its own cost and benefit. However, in terms of blocking probability and utilization achieved, any such option will perform no worse than the case of no wavelength conversion and no better than 


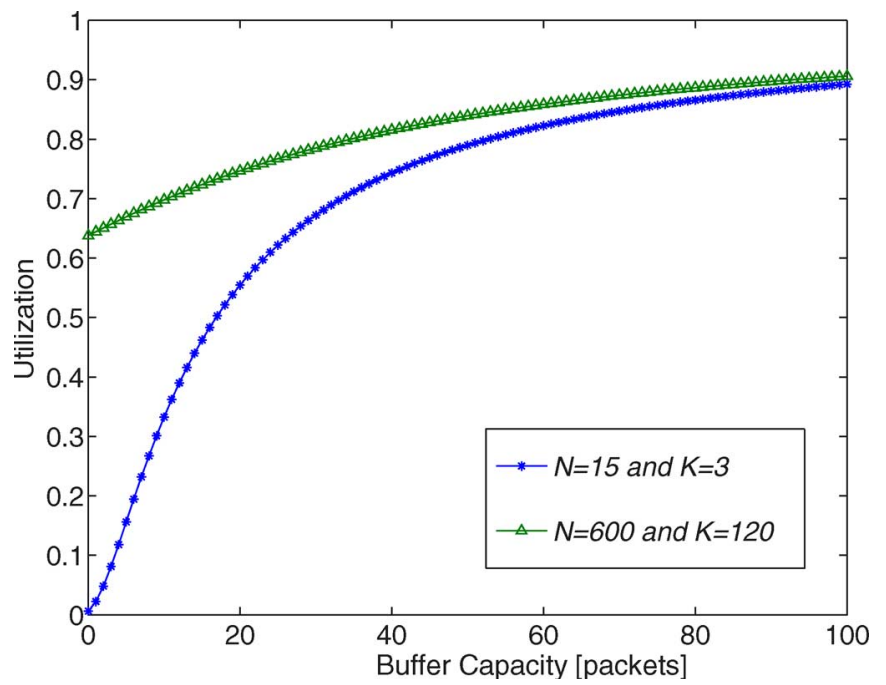

Fig. 8. Utilization versus buffer capacity [packets] for $\mathrm{M} / \mathrm{M} / K / K+B$, with $P_{B}=10^{-6}$.

the case of full wavelength conversion. The exact performance curves of various options of limited wavelength conversion is beyond the scope of this paper.

\section{CONCLUSION}

We have considered an open-loop model of a single-node optical packet switch and provided means for evaluation of packet blocking probability and mean queueing delay. Although in bufferless cases without wavelength conversion we may need to sacrifice too much bandwidth or, in other words, run the transmission links almost empty to obtain acceptable levels of blocking probability, a small amount of buffering can improve utilization markedly. This indicates that optical buffers may not need to be as large as previously thought. However, it is premature to make strong conclusions about the potential of all-optical switching. It is expected that, without wavelength conversion, efficiency on routes with many hops in an alloptical network will be significantly reduced. Electronically switched networks, in fact, provide full wavelength conversion for free, and this is a considerable advantage. Furthermore, the concerns raised in [34] regarding the power requirements of high-capacity optical switches and the technological advances in electronics should always be considered when the potential of OPS commercialization is evaluated.

\section{APPENDIX}

From (10), the average packet blocking probability $P_{B}$ is given by

$$
P_{B}=1-\frac{C}{O}
$$

or

$$
P_{B}(x)=1-\frac{\sum_{i=0}^{K+B-1}(N-i) \frac{x}{\mu} p_{i}}{\sum_{i=0}^{K+B}(N-i) \frac{x}{\mu} p_{i}} .
$$

We can rewrite $(N-i) x p_{i} / \mu$ as $a_{i} x^{i}$, where $a_{i}(>0)$ is a constant. Therefore, we can rewrite $P_{B}(x)$ as

$$
\begin{aligned}
P_{B}(x) & =\frac{a_{K+B} x^{K+B}}{\sum_{i=0}^{K+B} a_{i} x^{i}} \\
& =\frac{1}{1+\left(\sum_{i=0}^{K+B-1} a_{i} x^{i}\right) /\left(a_{K+B} x^{K+B}\right)} \\
& =\frac{1}{1+\sum_{i=0}^{K+B-1} \frac{1}{f_{i}}}
\end{aligned}
$$

where

$$
f_{i}=\frac{a_{K+B}}{a_{i}} x^{K+B-i}
$$

Taking the derivative of $P_{B}(x)$ against $x$, we have

$$
\frac{d P_{B}(x)}{d x}=\frac{\sum_{i=0}^{K+B-1} \frac{1}{f_{i}^{2}} \frac{d f_{i}}{d x}}{\left(1+\sum_{i=0}^{K+B-1} \frac{1}{f_{i}}\right)^{2}} .
$$

Then, we take the derivative of $f_{i}$ against $x$ and have

$$
\frac{d f_{i}}{d x}=(K+B-i) \frac{a_{K+B}}{a_{i}} x^{K+B-i-1}>0 .
$$

Note that $K+B-i>0$. Therefore, we obtain $d P_{B}(x) / d x>$ 0 . This means that $P_{B}(x)$ is a monotonically increasing function of $x$.

\section{ACKNOWLEDGMENT}

The authors would like to thank Z. Rosberg and A. Zalesky for their valuable discussions on the uniqueness and convergence of fixed-point solutions and R. Tucker for reviewing and commenting on the manuscript.

\section{REFERENCES}

[1] D. J. Blumenthal, P. R. Prucnal, and J. R. Sauer, "Photonic packet switches: Architectures and experimental implementations," Proc. IEEE, vol. 82, no. 11, pp. 1650-1667, Nov. 1994.

[2] D. J. Blumenthal, R. J. Feuerstein, and J. R. Sauer, "First demonstration of multihop all-optical packet switching," IEEE Photon. Technol. Lett., vol. 6, no. 3, pp. 457-460, Mar. 1994.

[3] D. K. Hunter, M. C. Chia, and I. Andonovic, "Buffering in optical packet switches," J. Lightw. Technol., vol. 16, no. 12, pp. 2081-2094, Dec. 1998.

[4] P. Gambini et al., "Transparent optical packet switching: network architecture and demonstrators in the KEOPS project," J. Sel. Areas Commun., vol. 16 , no. 7 , pp. $1245-1259$, Sep. 1998.

[5] C. Guillemot et al., "Transparent optical packet switching: The European ACTS KEOPS project approach," J. Lightw. Technol., vol. 16, no. 12, pp. 2117-2134, Dec. 1998

[6] D. Chiaroni, "Physical and logical validation of a network based on alloptical packet switching systems," J. Lightw. Technol., vol. 16, no. 12, pp. 2255-2264, Dec. 1998.

[7] S. Yao, B. Mukherjee, and S. Dixit, "Advances in photonic packet switching: An overview," IEEE Commun. Mag., vol. 38, no. 2, pp. 84-94, Feb. 2000.

[8] M. C. Chia et al., "Packet loss and delay performance of feedback and feed-forward arrayed-waveguide gratings-based optical packet switches with WDM inputs-outputs," J. Lightw. Technol., vol. 19, no. 9, pp. 1241-1254, Sep. 2001.

[9] L. Dittmann et al., "The European IST project DAVID: A viable approach toward optical packet switching," IEEE J. Sel. Areas Commun., vol. 21, no. 7, pp. 1026-1040, Sep. 2003.

[10] M. C. Yuang, P. L. Tien, and J. Shih, "QoS scheduler/shaper for optical coarse packet switching IP-over-WDM networks," IEEE J. Sel. Areas Commun., vol. 22, no. 9, pp. 1766-1780, Nov. 2004. 
[11] S. Y. Liew, G. Hu, and H. J. Chao, "Scheduling algorithms for shared fiber-delay-line optical packet switches-Part I: The single-stage case," $J$. Lightw. Technol., vol. 23, no. 4, pp. 1586-1600, Apr. 2005.

[12] S. Jiang et al., "Scheduling algorithms for shared fiber-delay-line optical packet switches-Part II: The three-stage clos-network case," J. Lightw. Technol., vol. 23, no. 4, pp. 1601-1609, Apr. 2005.

[13] I. Chlamtac and A. Fumagalli, "An optical switch architecture for Manhattan networks," IEEE J. Sel. Areas Commun., vol. 11, no. 4, pp. 550-559, May 1993.

[14] A. Ghafoor, M. Guizani, and S. Sheikh, "Architecture of an all-optical circuit-switched multistage interconnection network," IEEE J. Sel. Areas Commun., vol. 8, no. 8, pp. 1595-1607, Oct. 1990.

[15] A. Girard, Routing and Dimensioning in Circiuit-Switched Networks. Reading, MA: Addison-Wesley, 1990.

[16] R. Ramaswami and K. N. Sivarajan, "Routing and wavelength assignment in all-optical networks," IEEE/ACM Trans. Netw., vol. 3, no. 5, pp. 489-500, Oct. 1995.

[17] — , "Design of logical topologies for wavelength-routed optical networks," IEEE J. Sel. Areas Commun., vol. 14, no. 5, pp. 840-851, Jun. 1996.

[18] J. Turner, "Terabit burst switching," J. High Speed Netw., vol. 8, no. 1, pp. 3-16, Mar. 1999.

[19] C. Qiao and M. Yoo, "Optical burst switching (OBS): A new paradigm for an optical Internet," J. High Speed Netw., vol. 8, no. 1, pp. 69-84, Jan. 1999.

[20] S. Verma, H. Chaskar, and R. Ravikanth, "Optical burst switching: A viable solution for terabit IP backbone," IEEE Netw., vol. 14, no. 6, pp. 48-53, Nov./Dec. 2000.

[21] M. Yoo and C. Qiao, "Supporting multiple classes of services in IP over WDM networks," in Proc. GLOBECOM, Dec. 1999, vol. 1B, pp. 1023-1027.

[22] J. Wei and R. McFarland, "Just-in-time signaling for WDM optical burst switching networks," J. Lightw. Technol., vol. 18, no. 2, pp. 2019-2037, Dec. 2000.

[23] M. Yoo, C. Qiao, and S. Dixit, "Optical burst switching for service differentiation in the next-generation optical internet," IEEE Commun. Mag., vol. 39, no. 2, pp. 98-104, Feb. 2001.

[24] K. Dolzer, C. Gauger, J. Späth, and S. Bodamer, "Evaluation of reservation mechanisms for optical burst switching," AEÜ Int. J. Electron. Commun., vol. 55, no. 1, pp. 18-26, Jan. 2001.

[25] I. Baldine, G. N. Rouskas, H. G. Perros, and D. Stevenson, "JumpStart: A just-in-time signaling architecture for WDM burst-switched networks," IEEE Commun. Mag., vol. 14, no. 2, pp. 82-89, Feb. 2002.

[26] H. L. Vu and M. Zukerman, "Blocking probability for priority classes in optical burst switching networks," IEEE Commun. Lett., vol. 6, no. 5, pp. 214-216, May 2002.

[27] J. White, M. Zukerman, and H. L. Vu, "A framework for optical burst switching network design," IEEE Commun. Lett., vol. 6, no. 6, pp. 268-270, Jun. 2002.

[28] Z. Rosberg, H. L. Vu, M. Zukerman, and J. White, "Performance analyses of optical burst-switching networks," IEEE J. Sel. Areas Commun., vol. 21, no. 7, pp. 1187-1197, Sep. 2003.

[29] C. Cameron, H. Le Vu, J. Y. Choi, S. Bilgrami, M. Zukerman, and M. Kang, "TCP over OBS-Fixed-point load and loss," Opt. Express, vol. 13, no. 23, pp. 9167-9174, Nov. 2005.

[30] N. Barakat and E. H. Sargent, "Dual-header optical burst switching: A new architecture for WDM burst-switched networks," in Proc. IEEE INFOCOM, Mar. 2005, vol. 1, pp. 685-693.

[31] _ - "Analytical modeling of offset-induced priority in multiclass OBS networks," IEEE Trans. Commun., vol. 53, no. 8, pp. 1343-1352, Aug. 2005.

[32] R. S. Tucker, P. C. Ku, and C. J. Chang-Hasnain, "Delay-bandwidth product and storage density in slow-light optical buffers," Electron. Lett., vol. 41, no. 4, pp. 208-209, Feb. 2005.

[33] R. S. Tucker, P.-C. Ku, and C. J. Chang-Hasnain, "Slow-light optical buffers: capabilities and fundamental limitations," J. Lightw. Technol., vol. 23 , no. 12 , pp. 4046-4066, Dec. 2005.

[34] R. S. Tucker, "Peta bit-per-second routers: Optical vs. electronic implementations," presented at the Optical Fiber Conf., Anaheim, CA, Mar. 2006, Paper OFJ3.

[35] M. Enachescu, Y. Ganjali, A. Goel, T. Roughgarden, and N. McKeown, "Part III: Routers with very small buffers," ACM/SIGCOMM Comput. Commun. Rev., vol. 35, no. 3, pp. 83-89, Jul. 2005.

[36] ㄴ. (2005). "Part III: Routers with very small buffers," Stanford Univ., High Performance Networking Group, Stanford, CA. Stanford HPNG Tech. Rep. TR05-HPNG-060606. [Online]. Available: http://yuba. stanford.edu/yganjali/research/publications/TR05-HPNG-060606.pdf

[37] L. Breslau, D. Estrin, K. Fall, S. Floyd, J. Heidemann, A. Helmy, P. Huang, S. McCanne, K. Varadhan, Y. Xu, and H. Yu, "Advances in net- work simulation," IEEE Computer, vol. 33, no. 5, pp. 59-67, May 2000. [Online]. Available: http://www.isi.edu/johnh/PAPERS/Bajaj99a.html

[38] G. Appenzeller, I. Keslassy, and N. McKeown, "Sizing router buffers," in Proc. ACM SIGCOMM, New York, 2004, pp. 281-292.

[39] L. L. H. Andrew, T. Cui, J. Sun, M. Zukerman, K.-T. Ko, and S. Chan, "Buffer sizing for nonhomogeneous TCP sources," IEEE Commun. Lett., vol. 9, no. 6, pp. 567-569, Jun. 2005.

[40] T. Engset, "Die wahrscheinlichkeitsrechnung zur bestimmung der wahleranzahl in automatischen fernsprechamtern," Elektrotechnische zeitschrift, vol. 39, no. 31, pp. 304-306, Aug. 1918.

[41] A. Detti, V. Eramo, and M. Listanti, "Performance evaluation of a new technique for IP support in a WDM optical network: Optical composite burst switching (OCBS)," J. Lightw. Technol., vol. 20, no. 2, pp. 154-165, Feb. 2002.

[42] M. Zukerman, E. W. M. Wong, Z. Rosberg, G. M. Lee, and H. L. Vu, "On teletraffic application to OBS," IEEE Commun. Lett., vol. 8, no. 2, pp. 116-118, Feb. 2004.

[43] H. Overby. (2005, Mar.). "Performance modelling of optical packet switched networks with the Engset traffic model," Opt. Express, vol. 13, no. 5, pp. 1685-1695. [Online]. Available: http://www.opticsexpress.org/ abstract.cfm?URI $=$ OPEX-13-5-1685

[44] H. L. Vu, A. Zalesky, E. W. M. Wong, Z. Rosberg, S. M. H. Bilgrami, M. Zukerman, and R. S. Tucker, "Scalable performance evaluation of a hybrid optical switch," J. Lightw. Technol., vol. 23, no. 10, pp. 2961-2973, Oct. 2005.

[45] E. W. M. Wong and M. Zukerman. (2005, Nov.). "Performance evaluation for an optical hybrid switch with circuit queued reservations," Opt. Express, vol. 13, no. 23, pp. 9446-9459. [Online]. Available: http://www.opticsexpress.org/abstract.cfm?URI = OPEX-13-23-9446

[46] - "Analysis of an optical hybrid switch," IEEE Commun. Lett., vol. 10, no. 2, pp. 108-110, Feb. 2006.

[47] J. Hui, Switching and Traffic Theory for Integrated Broadband Networks. Norwell, MA: Kluwer, 1990

[48] L. Kleinrock, Queueing Theory, vol. 1. Hoboken, NJ: Wiley, 1975.

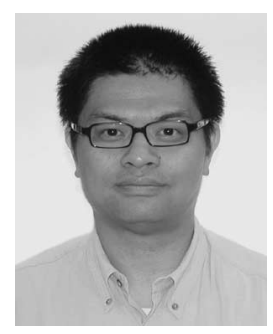

Eric W. M. Wong (S'87-M'90-SM'00) received the B.Sc. and M.Phil. degrees in electronic engineering from the Chinese University of Hong Kong, Hong Kong, in 1988 and 1990, respectively, and the Ph.D. degree in electrical and computer engineering from the University of Massachusetts, Amherst, in 1994.

In 1994, he joined the City University of Hong Kong, where he is now an Associate Professor with the Department of Electronic Engineering. His most notable research work involved the first workable model on state dependent dynamic routing. Since 1991, the model has been used by AT\&T to design and dimension its telephone network using real-time network routing. His research interests are in the analysis and design of telecommunications networks, optical burst switching, and video-on-demand.

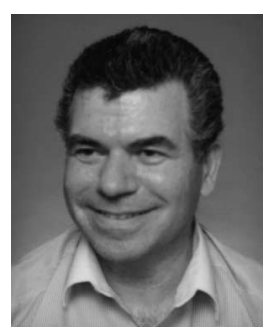

Moshe Zukerman (M'87-SM'91) received the B.Sc. degree in industrial engineering and management and the M.Sc. degree in operations research from Technion-Israel Institute of Technology, Haifa, Israel, and the $\mathrm{Ph} . \mathrm{D}$. degree in electrical engineering from University of California, Los Angeles, in 1985.

He was an independent Consultant with the IRI Corporation and a Postdoctoral Fellow with the University of California, Los Angeles, in 1985-1986. In 1986-1997, he was with the Telstra Research Laboratories (TRL), first as a Research Engineer and, in 1988-1997, as a Project Leader. He also taught and supervised graduate students at Monash University in 1990-2001. In 1997, he joined The University of Melbourne, Victoria, Australia, where he is now a Professor, responsible for promoting and expanding telecommunications research and teaching in the Electrical and Electronic Engineering Department. He has over 200 publications in scientific journals and conference proceedings. He coauthored two award-winning conference papers.

Dr. Zukerman was the recipient of the Telstra Research Laboratories Outstanding Achievement Award in 1990. He served on the editorial board of the Australian Telecommunications Research Journal, Computer Networks, and IEEE Communications Magazine. He also served as a Guest Editor of IEEE Journal of Selected AREas in Communications for two issues. Currently, he is serving on the editorial board of the IEEE/ACM TRANSACTIONS ON NETWORKING and the International Journal of Communication Systems. 